\title{
SEM and ToF-SIMS Ion Imaging Applied to Characterization of Fungal Biodeterioration of Paper in the Context of Cultural Heritage Collections
}

\author{
Hanna M. Szczepanowska ${ }^{1}$ and Yulia S. Goreva ${ }^{2}$ \\ 1. Museum Conservation Institute, Smithsonian Institution, Suitland MD, USA \\ 2. Mineral Sciences Department, Smithsonian Institution, Washington DC, USA
}

Fungal biodeterioration of artworks on paper and historic documents results from complex interactions of heterogenous cellular materials with living systems. The biodeterioration of paper is manifested by prominent staining and structural polymer degradation of paper by fungal enzymatic activities. Pigmentation produced by fungi results from their complex bio-chemical processed within their cell structures and is defined as secondary naphtoquinon metabolites. Although fungal biodeterioration has been studied for number of years the research has been focused on visual effects of biodeterioration, identification and description of fungal species, stipulation of causes and DNA sequencing; no studies of interfaces of fungi and paper matrix have been undertaken. Furthermore, black stains on paper caused by pigmented mycelium and spores only recently received some attention [1] most studies were focused on black staining of stone sculptures [2, 3]. This investigation explores stain formation attributed to meristematic fungi and employs time-of-flight secondary ionization mass spectrometry (ToF-SIMS) to chemically characterize biodeteriorated paper.

Materials and methods. Two historic papers affected by black fungal stains were used in this study, a 17th century study sheet from the collection of Maltese Archives, Malta, and 1920 Engraving on paper. Total number of stains tested was 26 and 14 on each paper respectively. The fiber composition of both papers was cotton. Low-vacuum environmental SEM Hitachi S 3700N was used for back scattered electron imaging $(12-15 \mathrm{kV})$. Spectral analysis and imaging was performed using a ToF-SIMS IVt (ION-TOF GmbH) time of flight secondary ion mass spectrometer with a $25 \mathrm{keV} \mathrm{Bi}_{3}{ }_{3}$ beam (pulsed current of $0.3 \mathrm{pA}$ ) rastered over the areas of $200 \times 200 \mu \mathrm{m}$ for $\sim 300 \mathrm{sec}$. The accumulated primary ion dose never exceeded $1.25 \times 10^{10}$ ions per $\mathrm{m}^{2}$ (below the static limit). Analyses were done with the instrument optimized for high spatial resolution (burst alignment mode). Chemical images were generated by collecting a mass spectrum at every pixel as the primary ion beam was rastered across the sample surface.

Results and discussion. SEM image (Figure 1B) shows morphology of fungal thick-walled spores in aggregates and chain formations. Fungal spores appear to be attached to the paper fibers both on the surface and inside the paper matrix. Comparison of ToF-SIMS spectra of heavily stained area and a clean area of the paper revealed that the stained part is rich in N-bearing organic compounds, likely of fungal origin. Figure 2 is ToF-SIMS ion images of the stained areas showing distribution of N-bearing compounds (A) and cellulose (B). Figure $2 \mathrm{C}$ is an overlay of cellulose with chemical signature of fungi. From textural relationships, one may conclude that fungi cover most of cellulose fibers in the analyzed area, but not all. Fungal mat is underneath of 2 cellulose fibers (top and bottom of the scanned area), thus confirming SEM finding that fungi grew into the matrix of paper yet remained mostly attached to the surface of paper fibers.

Our preliminary data shows that ToF-SIMS might become a valuable technique in defining the relationship between fungal growth and paper substrate. Our further work will involve using ToF-SIMS for chemical characterization of various (especially black and brown) pigments, finding their chemical signatures, and imaging their relationship with a paper substrate. The findings contribute to a better 
understanding of fungi and paper interactions carrying implication for the preservation of paper-based cultural heritage material and elucidating difficulties of removing bio-deposits from paper [4].

\section{References:}

[1] H M Szczepanowska, Th Mathia and P Belin. Morphology of fungal stains on paper characterized with multi-scale and multi-sensory surface metrology. Scanning 36 (2013), p.76-85.

[2] K Sterflinger and W E Krumbein. Dematiaceous fungi as a major agent for biopitting on Mediterranean marbles and limestones. Geomicrobiological Journal 14 (1997), p.219-230.

[3] C Urzi et al. Biodiversity of the rock inhabiting microflora with special reference to black fungi and black yeasts. In Microbial Diversity and Ecosystem Function, eds Allsopp, D Colwell, R R and D L Hawksworth, (1995), p. 289-302.

[4] Yulia Goreva acknowledges funding from the Deep Carbon Observatory.
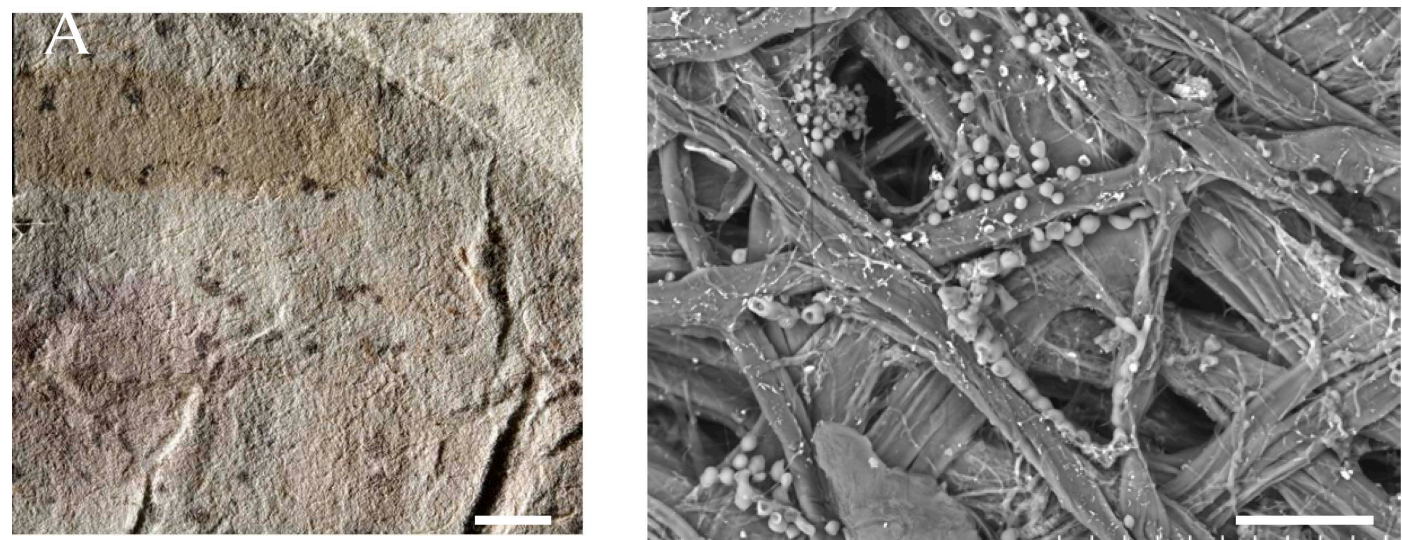

Figure 1. The $17^{\text {th }} \mathrm{c}$. paper from the Maltese Archives disfigured by black fungal stains was used in this investigation. A) Optical image of a paper fragment, scale bar $=10 \mathrm{~mm}$. B) SEM-VP micrograph of the black fungal stain shows morphology of spores visible as semi-spherical cells deposited in the fibrous paper substrate. Scale bar $=40 \mu \mathrm{m}$.
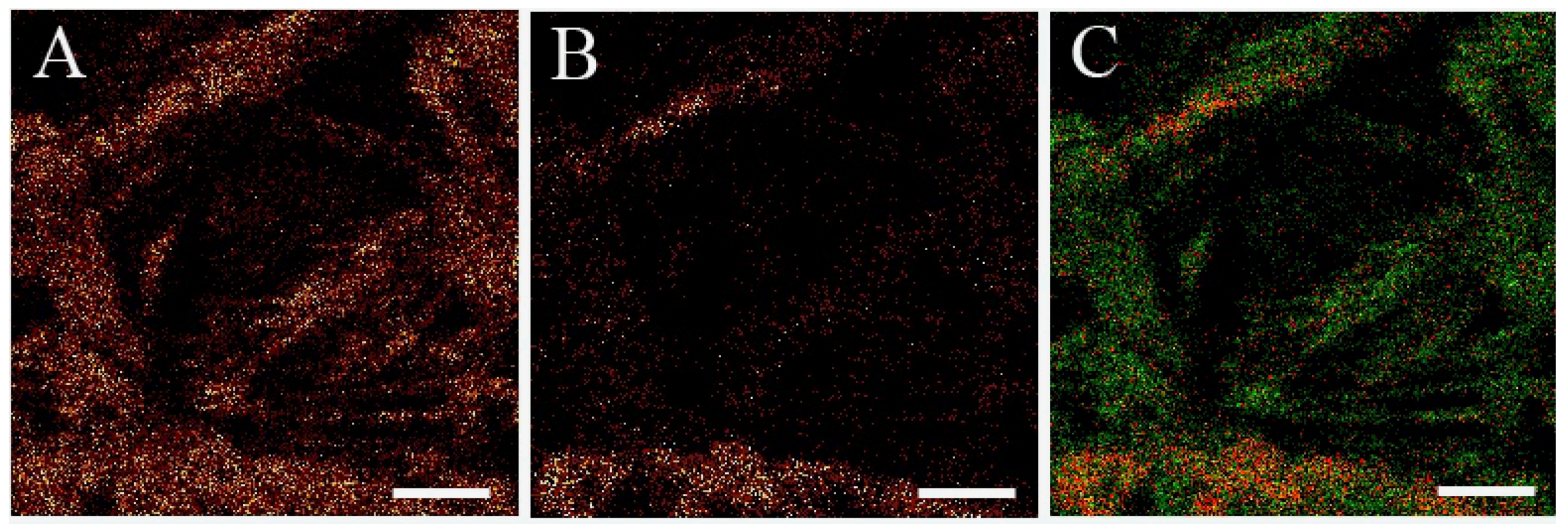

Figure 2. ToF-SIMS ion images of a paper fragment shown in Fig.1. A) Composite ion image of co-localized $\mathrm{N}$-containing organic compounds. B) Ion image of $\mathrm{C} 3 \mathrm{H} 7 \mathrm{O}+$ (an organic fragment of cellulose). C) Color overlay of in A (green) and B (red). White scale bars $=40 \mu \mathrm{m}$. 